\title{
Neuro-telehealth for fragile patients in a tertiary referral neurological institute during the COVID-19 pandemic in Milan, Lombardy
}

\author{
Davide Pareyson ${ }^{1,2}$ (D) $\cdot$ Chiara Pantaleoni ${ }^{1} \cdot$ Roberto Eleopra $^{1} \cdot$ Giuseppe De Filippis ${ }^{1} \cdot$ Isabella Moroni ${ }^{1} \cdot$ Elena Freri $^{1}$. \\ Federica Zibordi ${ }^{1}$ - Sara Bulgheroni ${ }^{1}$ - Emanuela Pagliano ${ }^{1}$. Daniela Sarti ${ }^{1}$ - Antonio Silvani ${ }^{1}$ - Licia Grazzi ${ }^{1}$. \\ Pietro Tiraboschi ${ }^{1}$. Giuseppe Didato ${ }^{1}$. Elena Anghileri ${ }^{1}$ - Anna Bersano ${ }^{1}$ - Laura Valentini ${ }^{1}$ - Sylvie Piacentini ${ }^{1}$. \\ Cristina Muscio ${ }^{1}$ - Matilde Leonardi ${ }^{1}$. Caterina Mariotti ${ }^{1}$ - Marica Eoli ${ }^{1}$. Sara Nuzzo ${ }^{1} \cdot$ Fabrizio Tagliavini $^{1}$. \\ Paolo Confalonieri ${ }^{1}$ • Francesca De Giorgi ${ }^{1}$ • for the Besta-Telehealth Task Force
}

Received: 23 February 2021 / Accepted: 10 April 2021 / Published online: 30 April 2021

(C) Fondazione Società Italiana di Neurologia 2021

\begin{abstract}
Background Lombardy was severely hit by the COVID-19 pandemic since February 2020 and the Health System underwent rapid reorganization. Outpatient clinics were stopped for non-urgent patients: it became a priority to manage hundreds of fragile neurological patients who suddenly had less reference points. In Italy, before the pandemic, Televisits were neither recognized nor priced.

Methods At the Fondazione IRCCS Istituto Neurologico C. Besta, we reorganized outpatient clinics to deliver Neurotelemedicine services, including Televisits and Teleneurorehabilitation, since March 2020. A dedicated Working Group prepared the procedure, tested the system, and designed satisfaction questionnaires for adults and children.

Results After a pilot phase, we prepared a procedure for Telemedicine outpatient clinics which was approved by hospital directions. It included prescription, booking, consenting, privacy and data protection, secure connection with patients (Teams Microsoft 365), electronic report preparation and delivery, reporting, and accountability of the services. During the MarchSeptember 2020 period, we delivered 3167 Telemedicine services, including 1618 Televisits, to 1694 patients (972 adults, 722 children) with a wide range of chronic neurological disorders. We successfully administered different clinical assessment and scales. Satisfaction among patients and caregivers was very high.

Conclusions During the dramatic emergency, we were able to take care of more than 1600 patients by organizing Neurotelehealth in a few weeks, lessening the impact of the pandemic on fragile patients with chronic neurological disorders; this strategy is now stably embedded in our care pathways. In Italy, Telehealth is at present recognized and priced and is becoming a stable pillar of the health system.
\end{abstract}

Keywords Neuro-telehealth $\cdot$ Televisit $\cdot$ Telemedicine $\cdot$ Teleneurorehabilitation $\cdot$ COVID-19 $\cdot$ Neurology

\section{Introduction}

The COVID-19 emergency required fast responses to prevent serious health consequences for fragile patients with chronic diseases. In March 2020, Lombardy became the centre of the

Davide Pareyson

davide.pareyson@ istituto-besta.it

Fondazione IRCCS Istituto Neurologico Carlo Besta, Milan, Italy

2 Rare Neurodegenerative and Neurometabolic Diseases Unit, Clinical Neurosciences Department, Fondazione IRCCS Istituto Neurologico Carlo Besta, Via Celoria 11, 20133 Milan, Italy
SARS-CoV2 epidemic in Italy. Since the 8th of March, during the lockdown period, people were confined at home and could go out only for important and proven needs. Fondazione IRCCS Istituto Neurologico Carlo Besta is a research institute placed in Milan, providing care to adult patients and children with neurological and neurosurgical diseases coming from all regions of Italy. Many of our patients are frail patients, affected by rare neurological disorders and/or by diseases with great complexity, requiring monitoring and follow-up visits as well as high specialization for proper management, and about $60 \%$ of them live outside Lombardy. The Lombardy Health Regional System suspended all non-urgent medical visits to concentrate efforts on fighting COVID-19 in several hospitals and to avoid the spreading of the contagion to patients, 
caregivers, and health-system personnel. During the first wave, hospitals were a place of diffusion of the disease and many health-workers became infected.

Although our Institute was identified by Regional health authorities as a COVID-19-free hospital and a Hub for Neurology and Neurosurgery for non-COVID-19 patients (as it has no emergency department and is composed of Neurological, Neurosurgical, Child Neurology, and Research Departments), a proportion of admitted patients in the first weeks of the epidemic proved to be SARS-CoV2 positive. Some of our physicians and nurses were seconded to other COVID-19 hospitals to help, whereas others became infected with SARS-CoV2.

About 2000 planned neurological visits were cancelled between March and May 2020, in most cases by the patients themselves, who feared to become infected during the travel to the hospital and within the hospital, or lived far away from Milan and had difficulties in travelling across Italy. We therefore needed a solution for continuing to provide care to many fragile patients with chronic neurological diseases and developed a fast and comprehensive Telehealth system.

Telehealth was still not recognized as a mean to provide medical care in our National Health System. In 2014, the Italian Health Ministry issued a document for providing address lines for Telehealth development [1]. However, since then, no formal decision had been taken and until March 2020, there was no formal recognition and no reimbursement for clinical services such as Televisits and Teleconsultations. Therefore, there were many different problems to be solved to provide care using Telehealth to our patients. During the epidemic, on the 18th of March 2020, the Lombardy Region issued a circular to allow delivery of all mental health services for paediatric patients and their families remotely by voice or video-call, using protected video conferencing platforms in accordance with the Decree of the General Director of the Welfare DG of Lombardy Region N. 2906 of the 8th of March and N. 3553 of the 15th of March 2020 [2], in addition to the national recommendation of Istituto Superiore di Sanità in Italy [3]. These were important steps and we kept such guidelines as the basis for developing Telehealth in our Institute during the COVID-19 emergency phase. In particular, all the Units of the Department of Paediatric Neuroscience (DINEP) were largely reorganized to maintain continuity of care and manage the new needs of families and of children that were also at home from schools [4-6].

\section{Material and methods}

Since 2018, we had already designed a scientific project to set the proper conditions for Telehealth and to deliver Televisits, Teleconsultations, and Telemonitoring in a clinical research setting for taking care of patients with different neurological disorders. We had analyzed and proposed solutions to delicate issues such as privacy, data safety, legal responsibilities, reporting, and charging. However, we had no information system in place for delivering Televisits and Teleconsultations, yet, as its development was part, still not carried out, of the research project.

We therefore had to organize the booking, the Televisit with a protected connection, and the reporting phase.

We set up a working group composed of the IT Office Chief (FDG) and four clinicians interested in Telehealth development: one expert in rare neurological diseases (DP), one in Parkinson disease (RE), one in multiple sclerosis (PC), and one in child neurology (CP). The group interacted with the booking office. We had full support from all the management (scientific director, general and health managers, president) of our Institute. We obtained the endorsement from the Health Ministry and informed the Lombardy Region of our project. No ethics board approval was needed as the Telehealth development was part of the normal clinical practice.

We set up an agreement to use Teams as a protected connection with patients. Teams is an app of Microsoft Office 365, compliant with General Data Protection Regulation (GDPR), for which our Institute had already a licence for use.

We started in mid-March with a 2-week pilot phase in the four Units and thereafter diffused the Televisits to all Units of Neurology, Child Neurology, and also of Neurosurgery.

We prepared two online questionnaires (one for adults and one for children) to be completed by the patients after the Televisits. To assess the level of satisfaction with Telemedicine for children and parents/carers, defined as the match between expected care and received care, we used the Telemedicine Satisfaction Questionnaire (TSQ), a 14-item survey scored on a five-point scale that assesses satisfaction and willingness to use Telehealth technology in the future [7]; it was translated to Italian and adapted to consent the rating by the patient's carers and not by the patient herself/himself [6], and administered to a subset of 75 carers after the second half of May 2020. The Questionnaire for adults included 47 questions related to patients' and caregivers' satisfaction as well as to technical issues and visit efficacy; we used the TSQ [7] and the client satisfaction questionnaire (CSQ) [8] as bases for selecting or designing the questions. It was administered to a subset of 78 patients with rare neurological diseases and multiple sclerosis. CSQ is validated in Italian whereas we did not have the chance to formally validate the TSQ in Italian, which is a clear limitation but in our opinion does not change the general meaning of the patients' and caregivers' opinion results.

\section{Results}

\section{Organization of Neuro-telehealth}

A procedure, in line with national and international guidelines/recommendations [1-3, 9-13], was defined for 
the neurological, neurosurgical, and neuropaediatric visits and was formally approved by the Institute Directions. We called all the patients who had a follow-up visit booked during the 23rd of February-31st of May period and proposed a Televisit for those able to use a computer (alone or with the support of a caregiver) with internet connection and audio-visual equipment, and we offered the Televisits to patients who were booking visits after May 31. For those willing and able to perform the Televisit, we booked it through the usual booking system. Each patient received via email: (a) a leaflet explaining Televisit reasons and modality, (b) an informed consent leaflet about privacy and data treatment (as for inperson visits), (c) an informed consent to sign and send back for patients willing to receive the clinical report via email, and (d) instructions for connecting with Teams.

Before the Televisit, an invitation was sent through Teams to the patient email address with date/time of visit, and a link for the Teams protected connection.

During the day of the Televisit, the physician (or other health professional) used an individual Teams teleconference connection for each patient, employed the webcam and audio instruments of the computer to see and speak with the patient, who was connected from home (or from the general practitioner office) and was helped by relatives or caregivers if needed.

The physicians, whenever possible, used two monitors, one to see the patient and the other to write the report in the electronic clinical record and to see the clinical documents. The electronic report was prepared as for an in-person visit on the platform in use at our Institute (in accordance with the Digital Administration Code, legislative decree no. 82 of 7 March 2005, no. 179 of 22 August 2016 and no. 217 of 13 December 2017, and AGID, Agenzia per l'Italia Digitale, requirements, legislative decree no.178 of 29 September 2015). Each electronic report was labelled in the system as "Televisit". The report began with the statement that the Televisit was performed during the COVID-19 emergency by means of Teams platform. Each visit lasted about 30 min: 10' for clinical history and update from previous visit, 10 ' for the clinical examination through the webcam, 10' to discuss conclusions and therapy, and for therapy and exams prescriptions, if any. The report can be visualized on the regional system of electronic records if the patient is from Lombardy and has granted access for such consultation. Alternatively, the report was sent via email to patients providing written consent before the visit, protected by a password communicated to the patient through a different channel (e.g. orally during the Televisit). In all the other cases, the report was sent via regular mail or will be given to the patient at the next face-to-face visit.

The prescription of the visit was prepared electronically by the caring physician of our Institute with the label "Telemedicine", or collected by email from the patient if released by the general practitioner, before the visit for later accountability by the Administrative Office. The Booking Office marked the visit as "Televisit" with a specific electronic flag. At that period, the Televisits for adults had neither regulation nor pricing, so we kept trace of each Telemedicine service for future recognition by the Lombardy Region.

The same general procedure applied for Teleneurorehabilitation which however, as delivered only to children, had some peculiarities due to age and service provided.

\section{Number of visits and medical services during the lockdown period}

Overall, since the beginning of the pandemic until September 30, 3167 medical services were performed through Neurotelehealth by 88 health professionals ( 52 adult neurologists, 20 child neurologists, 2 neurosurgeons, 6 child psychologists, 3 language and speech therapists, and 5 neuropsychomototricists). We provided 1618 Televisits (follow-up neurological visits for adults and children, neurosurgical visits, genetic counselling services), 55 neuropsychological tests for adults, and 1494 other health services for children (clinical multidisciplinary and multidimensional assessment, psychological consultation or support, learning, language and speech rehabilitation, neurofunctional Telemonitoring, and parent coaching) (Table 1). Teleneurorehabilitation was activated for 53 children ( 27 patients with language or/and learning disorders and 26 patients with developmental motor disorders).

The majority of the Telemedicine services took place in the 16th of March-14th of June period, during the pandemic phase 1 (lockdown, until May 3) and phase 2 (less severe restrictions until June 14), when overall 2098 medical Teleservices (including 1124 Televisits) were carried out.

During the phase 3 , since June 15 th, many outpatient clinics resumed their full activities and patients often required to have in-person visits. However, Telehealth continued and 1069 other medical services were performed until September 30, of which 494 were Televisits.

Table 2 summarizes the characteristics of the patients with access to Telemedicine services and their diagnosis.

Table 1 Telemedicine services provided and demography of managed patients

\begin{tabular}{ll}
\hline Telemedicine services, total (n) & $\mathbf{3 1 6 7}$ \\
\hline - Televisits $(n)$ & 1618 \\
- Other services $(n)$ & 1549 \\
Managed patients (n) & $\mathbf{1 6 9 4}$ \\
Age, mean (SD, range)_years & $\mathbf{3 2 . 4}(\mathbf{2 2 . 5 , 0 - 9 7 )}$ \\
- Adults $(n)$ & 972 \\
- Children $(n)$ & 722 \\
- Females $(n)$ & 775 \\
- Males $(n)$ & 919 \\
\hline
\end{tabular}


We also administered different clinical scales, including: the adult myopathy assessment tool (AMAT), a scale for neuromuscular disorders assessing several motor tasks which do not require the direct presence of the examiner and can be delivered from distance [14], and the Spinal and Bulbar Muscular Atrophy Functional Rating Scale (SBMA-FRS), a specific scale for SBMA requiring the evaluation of ability of bulbar, trunk, and limb functions [15]; the UPDRS for Parkinson disease and the EDSS for multiple sclerosis with Telehealth adaptations [16, 17]; the Charcot-Marie-Tooth (CMT) Examination Scale (CMTES) for CMT patients [18], although we could not evaluate the sensory system and assessment of strength was possible for MRC values $\leq 3$ and more difficult for higher values.

The other health services for children and adolescents included remote clinical assessment and Telemonitoring parent using interview, checklist, and questionnaire regarding adaptive skills, behavioural and developmental functioning, and patient-reported neurofunctional questionnaires. We have performed also a store-and-forward method to upload videos of child's behaviours and interactions by parents/caregivers in order to enable a remote assessment and to share with parents/carers cognitive and motor rehabilitation suggestions. To protect the privacy of the patients, all videos were exchanged by means of a Sharepoint system, with regulated access. Sharepoint is an app of Microsoft Office 365, compliant with GDPR, for which our Institute had already a licence for use.

Telerehabilitation for scholar children with learning disabilities was performed with an online dyslexia platform (RIDInet, with Reading Trainer app) and editing audio-visual materials for dysorthography and dyscalculia (emplopying Teams); Telerehabilitation for pre-school children with language disorders was performed using an online platform (RIDInet with Speech app) and training with audio-visual materials (Teams).

\section{Satisfaction questionnaires}

Results from Satisfaction Neuro-telehealth questionnaires administered to a subset of patients are reported in Tables 3 (78 adults) and 4 (75 children parents/carers). Among adult patients, about $10 \%$ reported "few" $(5 \%)$ or "quite" $(5 \%)$
Table 2 Diagnoses of patients undergoing Televisits $(n=1618)$

\begin{tabular}{ll}
\hline Diagnoses & $n(\%)$ \\
\hline Departments of Clinical Neurosciences, of Diagnostics and Technology, and of Neurosurgery & 966 \\
Tumors & $161(16.6)$ \\
Cerebrovascular diseases & $53(5.5)$ \\
Headache & $120(12.4)$ \\
Chiari malformation and syringomyelia & $42(4.3)$ \\
Multiple sclerosis and related disorders & $77(8.0)$ \\
Neurogenetic and neuromuscular disorders* & $249(25.8)$ \\
Epilepsy and sleep disorders & $81(8.4)$ \\
Dementias & $55(5.7)$ \\
Parkinson and movement disorders & $93(9.6)$ \\
Miscellaneous & $35(3.6)$ \\
Department of Paediatric Neuroscience & 652 \\
Multiple sclerosis and related disorders & $10(1.5)$ \\
Tourette Syndrome and other movement disorders & $64(9.8)$ \\
Epilepsy & $195(29.9)$ \\
Neurogenetic and neuromuscular disorders** & $128(19.6)$ \\
Cerebral Palsy & $51(7.8)$ \\
Neurodevelopmental disorders*** & $90(13.8)$ \\
Language or/and learning disorders & $81(12.4)$ \\
Tumors and cerebrovascular disease & $14(2.2)$ \\
Neurocutaneous diseases & $11(1.7)$ \\
Craniostenosis syndrome and Chiari malformation & $8(1.2)$ \\
\hline
\end{tabular}

*Neuropathies, ataxias, spastic paraplegias, Huntington's chorea, mitochondrial and neurometabolic disorders, myopathies, myasthenia gravis, amyotrophic lateral sclerosis

**Neuropathies, ataxias, spastic paraplegias, mitochondrial and neurometabolic disorders, myopathies, myasthenia gravis, spinal muscular atrophy

***Genetic syndromes, global delay, intellectual disability, and autism spectrum disorders 


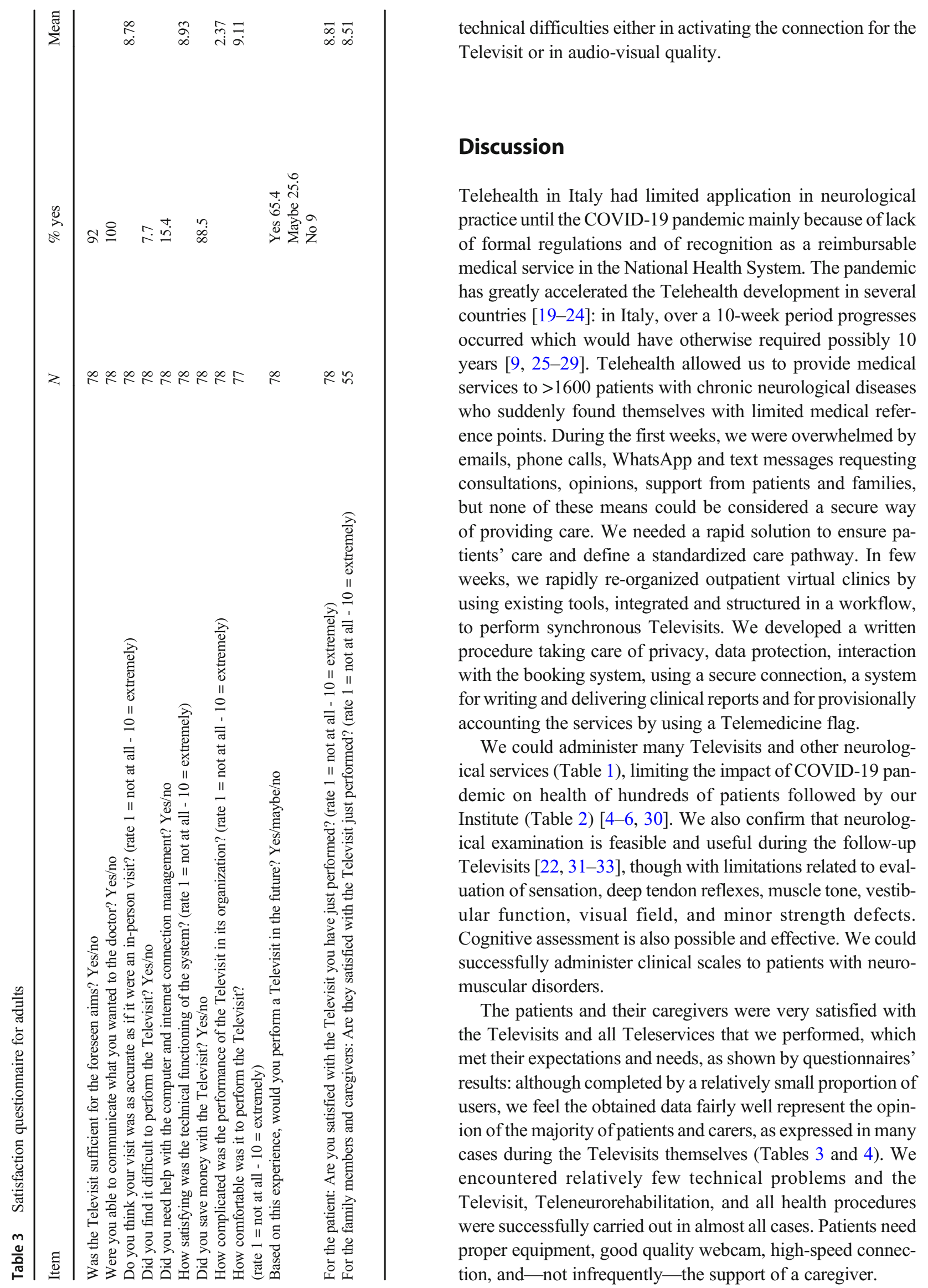

technical difficulties either in activating the connection for the Televisit or in audio-visual quality.

Telehealth in Italy had limited application in neurological practice until the COVID-19 pandemic mainly because of lack of formal regulations and of recognition as a reimbursable medical service in the National Health System. The pandemic has greatly accelerated the Telehealth development in several countries [19-24]: in Italy, over a 10-week period progresses occurred which would have otherwise required possibly 10 years $[9,25-29]$. Telehealth allowed us to provide medical services to $>1600$ patients with chronic neurological diseases who suddenly found themselves with limited medical reference points. During the first weeks, we were overwhelmed by emails, phone calls, WhatsApp and text messages requesting consultations, opinions, support from patients and families, but none of these means could be considered a secure way tients' care and define a standardized care pathway. In few to perform synchronous Televisits. We developed a written procedure taking care of privacy, data protection, interaction a system isionally We could administer many Televisits and other neurologservices (Table 1), limiting the impact of COVID-19 panby our neurologfollow-up evisits [22,31-33], though with limitations related to evalvestibfunction, visual field, and minor strength defects. Cognitive assessment is also possible and effective. We could uccessfully administer clinical scales to patients with neurodisorders.

The patients and their caregivers were very satisfied with he Televisits and all Teleservices that we performed, which met their expectations and needs, as shown by questionnaires' esults: although completed by a relatively small proportion of sers, we feel the obtained data fairly well represent the opinon of the majority of patients and carers, as expressed in many cases during the Televisits themselves (Tables 3 and 4). We ncountered relatively few technical problems and the Televisit, Teleneurorehabilitation, and all health procedures were successfully carried out in almost all cases. Patients need tion, and — not infrequently — the support of a caregiver. 
Table 4 Telemedicine Satisfaction Questionnaire (TSQ) for children

\begin{tabular}{lll}
\hline Item & $N$ & Mean* \\
\hline 1. I can easily talk to my healthcare provider. & 75 & 4.72 \\
2. I can hear my healthcare provider clearly. & 75 & 4.57 \\
3. My healthcare provider is able to understand the healthcare condition of my child. & 75 & 4.6 \\
4. I can see my healthcare provider as if we met in person. & 75 & 4.08 \\
5. I do not need assistance while using the system. & 75 & 4.63 \\
6. I feel comfortable communicating with my healthcare provider. & 75 & 4.67 \\
7. My child feels comfortable communicating with my healthcare provider. & 51 & 4.18 \\
8. I think the healthcare provided via Telemedicine is consistent. & 75 & 4.32 \\
9. I obtain better access to healthcare services by use of Telemedicine. & 75 & 3.57 \\
10. Telemedicine saves me time travelling to a hospital or specialist clinic. & 75 & 4.49 \\
11. I did receive adequate attention. & 75 & 3.84 \\
12. Telemedicine provides for the healthcare need of my child. & 75 & 4.74 \\
13. I find Telemedicine an acceptable way to receive healthcare services. & 75 & 3.93 \\
14. I will use Telemedicine services again. & 75 & 4.05 \\
15. Overall, I am satisfied with the quality of service being provided via Telemedicine. & 75 & 4.42 \\
\hline
\end{tabular}

*A 5-point scale was used with the following values: $1=$ strongly disagree, $2=$ somewhat disagree, $3=$ undecided, $4=$ somewhat agree, $5=$ strongly agree
Overall, Telehealth was very well received by doctors and also by other healthcare personnel, as confirmed by the high number of them involved in the process and by the number of Telemedicine services provided.

Limiting factors were lack of regulation and pricing of medical services, but the situation is rapidly changing and since December 2020 the Ministry of Health is recognizing and pricing Televisits [34]. There are still limitations in the use, for instance the first visits cannot be provided by Telehealth, and the Televisit can be used only for the follow-up of patients with an already diagnosed condition and with well-defined care pathways or needs [34]. However, a barrier has been definitively broken.

We strongly believe in the future of Telehealth and are convinced that many of our patients may benefit from Telemedicine services not only during the acute phases of the pandemic but also in the future: in particular, neurological patients needing tertiary neuro-care and living far away from hospital, with motor disability making it difficult to go to hospital, who maintain job activities, or who need frequent monitoring. Therefore, we have now a platform certified as a medical device according to the European Community requirements to provide Neurotelehealth and are stably delivering services of Televisits for adults and children and Teleneurorehabilitation for children, and we are organizing Telecooperation, Teleconsultation, Telemonitoring, to face the still ongoing pandemic phase and the post-COVID period. Although this paper reflects the experience of one Institute only, the global increased need for Telemedicine due to the pandemic requires that more and more case studies are reported, to show that despite the disruption of services there are ways in which health services can provide efficient mitigations for guaranteeing care for patients of any age and with any diagnosis.

Acknowledgements Besta-Telehealth Task Force, other participants Carlo Antozzi, Anna Ardissone, Enrica Bersano, Giorgio Boncoraglio, Salvatore Bonvegna, Andrea Botturi, Laura Brambilla, Laura Canafoglia, Luigi Caputi, Paola Caroppo, Maria Rita Carriero, Cecilia Casali, Marina Casazza, Alessia Catania, Claudia Ciaccio, Roberto Cilia, Eleonora Dalla Bella, Domenico D'Amico, Federica Rachele Danti, Stefano D'Arrigo, Marco De Curtis, Francesco Deleo, Grazia Devigili, Giuseppe Di Fede, Roberta Di Giacomo, Antonio Elia, Silvia Esposito, Margherita Estienne, Silvia Fenu, Mario Fichera, Gaetano Finocchiaro, Rita Frangiamore, Marta Gatti, Paola Gaviani, Giorgio Giaccone, Luca Giani, Anna Rita Giovagnoli, Nico Golfrè Andreasi, Tiziana Granata, Elisa Granocchio, Costanza Lamperti, Elena Lamperti, Massimo Leone, Riccardo Masson, Lorenzo Nanetti, Nardo Nardocci, Chiara Pastori, Chiara Pisciotta, Alberto Proietti Cecchini, Francesca Ragona, Veronica Redaelli, Veronica Saletti, Ettore Salsano, Emma Scelzo, Roberta Solazzi, Alessandra Tozzo, Susanna Usai, Giovanna Zorzi; Maria Teresa Arnoldi, Maria Foscan, Alessia Marchi, Ilaria Pedrinelli, Riccardo Zanin; Stefania Gazzola, Santina Magazù, Maria Rosa Scopelliti; Tiziana Casalino, Marinella De Salvatore, Sara Mazzanti, Matilde Taddei; Alessandro Fedeli; Davide Sattin; Luca Galimberti, Rocco Zagari, Marco Bombonato; Luigi Fonte; Simona Floridia

Funding This work is part of the Telehealth development project funded by Italian Ministry of Health (Ministero della Salute Progetto Telemedicina Conto Capitale: "Telemedicina per Ricerca Traslazionale e Continuità di Cura Sociosanitaria in Neurologia, Neuropediatria, Neuroriabilitazione e Neurochirurgia").

Availability of data (data transparency) Shared data are available as aggregate data upon motivated request to the corresponding author; they are deposited in the repository of reimbursement section of health services and of the health electronic records. 


\section{Declarations}

Conflict of interest The authors declare no competing interests.

Ethics approval No ethics board approval was needed as the Telehealth development was part of the normal clinical practice.

Informed consent All patients and carers provided written informed consent to the clinical services.

\section{References}

1. Italian Ministry of Health. National guidelines on telemedicine. 2014. Available at: http://www.salute.gov.it/imgs/C_17 pubblicazioni 2129 allegato.pdf (last accessed November $30^{-}$, 2020)

2. Decree of the General Director of the Welfare DG of Lombardy Region N. 2906 of March $8^{\text {th }}$ and N. 3553 of March $15^{\text {th }}$ 2020DGR Regione Lombardia, available at https://www.regione.lombardia.it/ wps/wcm/connect/5e0deec4-caca-409c-825b-25f781d8756c/ DGR $+2906+8+$ marzo+2020.pdf?MOD=AJPERES \&CACHEID $=$ ROOTWORKSPACE-5e0deec4-caca-409c-825b-25f781d8756c$\mathrm{n} 2 . \mathrm{vCsc}$ and https://www.regione.lombardia.it/wps/wcm/connect/ af6d2303-9212-433e-abcb-ac5a77b48fe5/Decreto+3353.pdf? MOD=AJPERES\&CACHEID=ROOTWORKSPACE-af6d23039212-433e-abcb-ac5a77b48fe5-n3DPIAT

3. Rapporto ISS COVID-19 n. 12/2020 - Indicazioni ad interim per servizi assistenziali di telemedicina durante l'emergenza sanitaria COVID-19. Versione del 13 aprile 2020: https://www.iss.it/ rapporti-covid-19/-/asset_publisher/btw1J82wtYzH/content/ rapporto-iss-covid-19-n.-12-2020-indicazioni-ad-interim-perservizi-assistenziali-di-telemedicina-durante-1-emergenzasanitaria-covid-19.-versione-del-13-aprile-2020

4. Granata T, Bisulli F, Arzimanoglou A, Rocamora R (2020) Did the COVID-19 pandemic silence the needs of people with epilepsy? Epileptic Disord 22:439-442. https://doi.org/10.1684/epd.2020. 1175

5. Sarti D, De Salvatore M, Gazzola S, Pantaleoni C, Granocchio E (2020) So far so close: an insight into smart working and telehealth reorganization of a Language and Learning Disorders Service in Milan during COVID-19 pandemic. Neurol Sci 41:1659-1662. https://doi.org/10.1007/s10072-020-04481-8

6. Taddei M, Bulgheroni S (2020) Facing the real time challenges of the COVID-19 emergency for child neuropsychology service in Milan. Res Dev Disabil 107:103786. https://doi.org/10.1016/j. ridd.2020.103786

7. Yip MP, Chang AM, Chan J, MacKenzie AE (2003) Development of the Telemedicine Satisfaction Questionnaire to evaluate patient satisfaction with telemedicine: a preliminary study. J Telemed Telecare 9:46-50. https://doi.org/10.1258/135763303321159693

8. Larsen DL, Attkisson CC, Hargreaves WA, Nguyen TD (1979) Assessment of client/patient satisfaction: development of a general scale. Eval Program Plann 2:197-207. https://doi.org/10.1016/ 0149-7189(79)90094-6

9. Stipa G, Gabbrielli F, Rabbito C, Di Lazzaro V, Amantini A, Grippo A, Carrai R, Pasqui R, Barloscio D, Olivi D, Lori S, Telemedicine Working Group (2020) The Italian technical/ administrative recommendations for telemedicine in clinical neurophysiology. Neurol Sci 24:1-9. https://doi.org/10.1007/s10072020-04732-8

10. Ferorelli D, Nardelli L, Spagnolo L, Corradi S, Silvestre M, Misceo F, Marrone M, Zotti F, Mandarelli G, Solarino B, Dell'Erba A (2020) Medical legal aspects of telemedicine in Italy: application fields, professional liability and focus on care services during the COVID-19 health emergency. J Prim Care Community Health 11: 2150132720985055. https://doi.org/10.1177/2150132720985055

11. Krupinski EA, Bernard J (2014) Standards and guidelines in telemedicine and telehealth. Healthcare (Basel) 2:74-93. https://doi. org/10.3390/healthcare2010074

12. AAN position statement on Telemedciine at https://www.aan.com/ policy-and-guidelines/policy/position-statements/telemedicine/

13. Free Webinar: Telemedicine and COVID-19 - American Academy of Neurology https://www.youtube.com/watch?v=Pw-Jdy3-T9g

14. Harris-Love MO, Fernandez-Rhodes L, Joe G et al (2014) Assessing function and endurance in adults with spinal and bulbar muscular atrophy: validity of the adult myopathy assessment tool. Rehabil Res Pract 2014:873872. https://doi.org/10.1155/2014/ 873872

15. Hashizume A, Katsuno M, Suzuki K et al (2015) A functional scale for spinal and bulbar muscular atrophy: cross-sectional and longitudinal study. Neuromuscul Disord 25:554-562. https://doi.org/10. 1016/j.nmd.2015.03.008

16. Schneider RB, Myers TL, Tarolli CG et al (2020) Remote administration of the MDS-UPDRS in the time of COVID-19 and beyond. J Parkinsons Dis 10:1379-1382. https://doi.org/10.3233/ JPD-202121

17. Kane RL (2008) Teleneurology in patients with multiple sclerosis: EDSS ratings derived remotely and from hands-on examination. Telemed Telecare 14:190-194. https://doi.org/10.1258/jtt.2008. 070904

18. Murphy SM, Herrmann DN, McDermott MP et al (2011) Reliability of the CMT neuropathy score (second version) in Charcot-Marie-Tooth disease. J Peripher Nerv Syst 16:191-198. https://doi.org/10.1111/j.1529-8027.2011.00350

19. Al Kasab S, Almallouhi E, Holmstedt CA (2020) Optimizing the use of teleneurology during the COVID-19 pandemic. Telemed J E Health 26:1197-1198. https://doi.org/10.1089/tmj.2020.0109

20. Bashshur R, Doarn CR, Frenk JM, Kvedar JC, Woolliscroft JO (2020) Telemedicine and the COVID-19 pandemic, lessons for the future. Telemed J E Health 26:571-573. https://doi.org/10. 1089/tmj.2020.29040.rb

21. Roy B, Nowak RJ, Roda R, Khokhar B, Patwa HS, Lloyd T, Rutkove SB (2020) Teleneurology during the COVID-19 pandemic: a step forward in modernizing medical care. J Neurol Sci 414: 116930. https://doi.org/10.1016/j.jns.2020.116930

22. Nitkunan A, Paviour D, Nitkunan T (2020) COVID-19: switching to remote neurology outpatient consultations. Pract Neurol 20:222 224. https://doi.org/10.1136/practneurol-2020-002571

23. Bertran Recasens B, Rubio MA (2020) Neuromuscular diseases care in the era of COVID-19. Front Neurol 11:588929. https://doi. org/10.3389/fneur.2020.588929

24. Spindler M, Jacobs D, Yuan K, Tropea T, Teng CW, Perrone C, Do D, Wechsler L (2020) A department approach to teleneurology. Telemed J E Health. https://doi.org/10.1089/tmj.2020.0323

25. Omboni S (2020) Telemedicine during the COVID-19 in Italy: a missed opportunity? Telemed J E Health 26:973-975. https://doi. org/10.1089/tmj.2020.0106

26. Cuffaro L, Di Lorenzo F, Bonavita S, Tedeschi G, Leocani L, Lavorgna L (2020) Dementia care and COVID-19 pandemic: a necessary digital revolution. Neurol Sci 41:1977-1979. https:// doi.org/10.1007/s10072-020-04512-4

27. Moccia M, Lanzillo R, Brescia Morra V, Bonavita S, Tedeschi G, Leocani L, Lavorgna L, Digital Technologies Web and Social Media Study Group of the Italian Society of Neurology (2020) Assessing disability and relapses in multiple sclerosis on tele-neurology. Neurol Sci 41:1369-1371. https://doi.org/10.1007/s10072020-04470-x

28. Bombaci A, Abbadessa G, Trojsi F, Leocani L, Bonavita S, Lavorgna L, Digital Technologies, Web and Social Media Study 
Group of the Italian Society of Neurology (2021) Telemedicine for management of patients with amyotrophic lateral sclerosis through COVID-19 tail. Neurol Sci 42:9-13. https://doi.org/10.1007/ s10072-020-04783-x

29. Iodice F, Romoli M, Giometto B, Clerico M, Tedeschi G, Bonavita S, Leocani L, Lavorgna L, Digital Technologies, Web and Social Media Study Group of the Italian Society of Neurology (2021) Stroke and digital technology: a wake-up call from COVID-19 pandemic. Neurol Sci 42:805-809. https://doi.org/10.1007/ s10072-020-04993-3

30. Cilia R, Mancini F, Bloem BR, Eleopra R (2020) Telemedicine for parkinsonism: a two-step model based on the COVID-19 experience in Milan, Italy. Parkinsonism Relat Disord 75:130-132. https://doi.org/10.1016/j.parkreldis.2020.05.038

31. Grossman SN, Han SC, Balcer LJ, Kurzweil A, Weinberg H, Galetta SL, Busis NA (2020) Rapid implementation of virtual neurology in response to the COVID-19 pandemic. Neurology 94: 1077-1087. https://doi.org/10.1212/WNL.0000000000009677
32. Saporta MA, Granit V, Lewis R, Benatar M (2020) Yes, we can: neuromuscular examination by telemedicine. Muscle Nerve 62: E83-E85. https://doi.org/10.1002/mus.27056

33. Robb JF, Hyland MH, Goodman AD (2020) Comparison of telemedicine versus in-person visits for persons with multiple sclerosis: a randomized crossover study of feasibility, cost, and satisfaction. Mult Scler Relat Disord 36:101258. https://doi.org/10.1016/j. msard.2019.05.001

34. Memorandum of Understanding between State and Regions, Legislative Decree no. 281 of 28 August 1997, repertoire of acts no.215/CRS of 17 December 2020 http://www.statoregioni.it/ media/3221/p-3-csr-rep-n-215-17dic2020.pdf

Publisher's note Springer Nature remains neutral with regard to jurisdictional claims in published maps and institutional affiliations. 